\title{
¿Es el dímero $D$ un marcador confiable de la letalidad durante la COVID-19 en Hispanoamérica?
}

\section{Is the D-dimer a reliable marker of fatality during COVID-19 in Latin America?}

Norma K. Delgado-Lavado ${ }^{1, a}$, Yomayra S. Chigne-Castro,a, Fernando E. EspinozaCueva $^{1, a}$, Christian E. Correa-Verde ${ }^{1, a}$, Esteban Y. Vergara-de la Rosa ${ }^{1, b}$

\begin{abstract}
Señor editor,
La pandemia causada por el SARS-CoV-2 presenta más de un millón de defunciones de los 28 millones de casos confirmados en todo el mundo. La gravedad de esta infección se determina usando tasa de letalidad, definida como el porcentaje del número de muertes por la infección respecto del total de casos COVID-19 confirmados. En Hispanoamérica, según la OMS, los seis pauses más afectados con mayor tasa de letalidad, son: México (10,54\%), Ecuador (8,59\%), Bolivia (5,85\%), Perú $(4,07 \%)$, Nicaragua $(3,67 \%)$ y Guatemala $(3,61 \%)^{(1)}$. Bajo este contexto, estos países utilizan el dímero D (DD) como marcador para la detección de eventos trombóticos, que son una de las complicaciones de la COVID-19 asociada a una probabilidad creciente de muerte intrahospitalaria ${ }^{(2)}$ con influencia en la letalidad.
\end{abstract}

El DD es un producto final de la degradación de un trombo rico en fibrina y se utiliza como marcador. El valor normal del DD es $\leq 0,5 \mu \mathrm{g} / \mathrm{ml}$ el cual nos indica baja probabilidad de desarrollar alguna coagulopatía, mientras que los valores elevados mayores a $1 \mu \mathrm{g} / \mathrm{ml}$ predicen posibles eventos trombóticos en pacientes hospitalizados por COVID-19(2). Existen hallazgos que correlacionan de forma positiva y creciente el nivel sérico del DD con la letalidad de la infección ${ }^{(3)}$, como se demuestra en un reporte, donde al $26,27 \%$ de pacientes hospitalizados se les encontró niveles de DD con una media de 5,2 $\mu \mathrm{g} / \mathrm{ml}$ y no llegaron a sobrevivir ${ }^{(2)}$.

Además de los niveles séricos del DD, existen otras propiedades como la especificidad, la sensibilidad, el valor predictivo (VP) y el tiempo de respuesta que se

\footnotetext{
. Facultad de Medicina, Universidad Nacional de Trujillo, Jr. Salaverry 545 , Trujillo, Perú. Estudiantes de pregrado.

Docente del departamento de Ciencias Básicas Médica.
}

pueden relacionar con la tasa de letalidad de la COVID19. Todas estas propiedades en los diferentes países se resumen en la Tabla 1.

Al inicio, se reconoció a la neumonía como patología principal de la COVID-19 ${ }^{(4)}$. Sin embargo, en esta infección se han ido manifestando otros cuadros clínicos como daños vasculares con eventos trombóticos (debido a hipoxia e inflamaciones), que se ubican en los estadios II (moderado) y III (grave) de la enfermedad $^{(5)}$. De acuerdo a esto, los países Hispanoamericanos, según su guía de manejo de pacientes contra la COVID-19, aplican el (DD) en las diferentes etapas para evitar mayores complicaciones (tabla 1 ). Por lo tanto, no existe un uso estandarizado del DD en estos países; por ejemplo, en México se le da uso en el estadio III; mientras que en Perú y Guatemala, en los estadios II y $\mathrm{III}^{(6-8)}$.

Por otra parte, la principal limitación de nuestro estudio fue considerar un tamaño de muestra que solo incluía a los seis primeros países Hispanoamericanos con una mayor tasa de letalidad. Además, hay una escasa evidencia de la posible relación directa que existe entre el uso de la prueba del dímero DD en las etapas II y III, y la tasa de letalidad por COVID-19, mostrado en la tabla 1, por lo que motivamos a los investigadores a realizar estudios con un mayor tamaño de muestra con el objetivo de verificar esta posible relación.

Dada la elevada cifra de fallecidos por COVID-19 en países Hispanoamericanos, debemos reconocer al DD como un importante marcador de eventos trombóticos que nos podría indicar una posible complicación de la infección. Asimismo, se podrían evitar mayores daños aplicando un tratamiento antitrombótico precoz a los pacientes con COVID-19 y de esta manera se reduciría la tasa de letalidad en cada uno de estos países. 
Tabla 1. Letalidad de la infección por COVID-19 asociada a propiedades y estadío en el que se usa el dímero D en seis países de Hispanoamérica ${ }^{(6-10)}$.

\begin{tabular}{|c|c|c|c|c|}
\hline \multirow{2}{*}{ PAÍSES HISPANOAMERICANOS } & \multirow{2}{*}{$\begin{array}{l}\text { ESTADÍO DE LA COVID-19 } \\
\text { EN EL QUE SE USA EL DD }\end{array}$} & \multicolumn{3}{|c|}{ Propiedades del DD } \\
\hline & & SENSIBILIDAD & ESPECIFICIDAD & TIEMPO DE RESPUESTA \\
\hline MÉXICO & III & Baja & Alta & Rápida (2-5minutos) \\
\hline ECUADOR & III & Baja & Alta & Rápida (2-5minutos) \\
\hline BOLIVIA & | y || & Baja & Alta & Rápida (2-5minutos) \\
\hline PERÚ & II y III & Baja & Alta & Rápida (2-5minutos) \\
\hline NICARAGUA & II y III & Alta & Baja & Lenta ( $2-4$ horas) \\
\hline GUATEMALA & II y III & Alta & Baja & Lenta (2-4 horas) \\
\hline
\end{tabular}

Conflictos de interés: Los autores niegan conflictos de interés.

Financiamiento: Autofinanciado.

\section{REFERENCIAS BIBLIOGRÁFICAS}

1. OMS. Panel de la OMS sobre la enfermedad por coronavirus (COVID-19) [Internet]. Panel de la OMS sobre la enfermedad por coronavirus (COVID19). 2020 [citado: 24 Setiembre 2020]. p. 1. Disponible en: https: / / covid19. who.int/

2. Zhou F, Yu T, Du R, Fan G, Liu Y, Liu Z et al. Clinical course and risk factors for mortality of adult inpatients with COVID-19 in Wuhan, China: a retrospective cohort study. The Lancet [Internet]. 2020 [ c i t a d o e l $100 \mathrm{ct} \mathrm{ubre}$ 2020];395(10229):1054-1062. Disponible en: https://www.thelancet.com/journals/lancet/art icle/PIIS0140-6736(20)30566-3/fulltext

3. Lima W, Barra A, Brito J, Niser W. D-Dimer serum levels as a biomarker associated for the lethality in patients with coronavirus disease 2019: a metaanalysis. Wolters Kluwer Health [Internet]. 2020 [citado el 10 Octubre 2020];31(5):335-337. D i s p o n i ble e $n$ : https://journals.lww.com/bloodcoagulation/Full text/2020/07000/d Dimer serum levels as_a b iomarker associated for.9.aspx

4. Jara F, Jimenez G, Sananay E, Murillo J, Molina P, Vélez J. Hipercoagulabilidad, trombosis intravascular y trombocitosis asociada al COVID19. Reporte de un caso. Bionatura [Internet]. 2020 [citado el 2 Octubre 2020];5(2):1138-1141. D i s p o n i b l e e n : http://revistabionatura.com/2020.05.02.11.htm $\underline{l}$

5. Siddiqi $\mathrm{H}$, Mehra M. COVID-19 illness in native and immunosuppressed states: A clinical-therapeutic staging proposal. J Heart Lung Transplant [Internet]. 2020 [citado el 2 Octubre
2020];39(5):405-407. Disponible en: https://www.ncbi.nlm.nih.gov/pmc/articles/PM C7118652/

6. Instituto Guatemalteco de Seguridad Social. Diagnóstico y manejo de pacientes sospechosos y confirmados de COVID-19. Ciudad de Guatemala: Subgerencia de Prestaciones en Salud; 2020 p. 20.

7. Elizalde J, Fortuna J, Luviano J, Mendoza V, Mijangos J, Olivares E et al. Guía COVID-19 para la atención del paciente crítico con infección por SARS-CoV-2 Colegio Mexicano de Medicina Crítica. México: Medicina Crítica; 2020 p. 31.

8. Ministerio de Salud del Perú. Documento Técnico: Manejo de personas afectadas por COVID-19 en áreas de atención crítica. Resolución Ministerial N² 254-2020-MINSA. Lima, Perú: MINSA; 2020

9. Linkins L, Lapner S. Review of D-dimer testing : Good, Bad, and Ugly. Rev Int Hematol Lab [Internet]. 2017 [citado el 2 Octubre 2020];39 (1) : $98-103$. D i s p o n ible e n : https://onlinelibrary.wiley.com/doi/full/10.111 1/ijlh.12665

10. Duboscq C, Ceresetto J, Martinuzzo M, Bottaro F, Ramos G, Echenagucia $M$ et al. Uso y limitaciones del dímero $D$ en la exclusión del tromboembolismo venoso. Revista Hematología [Internet]. 2018 [citado el 2 Octubre 2020];22 (1):55-65. D i s p o n i b l e e $n$ : http://www.revistahematologia.com.ar/index.p $\mathrm{hp} /$ Revista/article/view/112

\section{Correspondencia}

Norma Katherine Delgado Lavado

Dirección: Mz N3- Lt 18 Urb. Covicorti

Teléfono: 968943204

Correo: ndelgado@unitru.edu.pe

\section{Revisión de pares}

Recibido: 17/10/2020

Aceptado: 15/12/2020 